\title{
Extensive Audio-motor Experience Can Calibrate The Egocentric Space In Early Blindness
}

\author{
Davide Esposito ${ }^{1,2}$, Alice Bollini ${ }^{1}$, Monica Gori $^{1^{*}}$
}

${ }^{1}$ Unit for Visually Impaired People, Istituto Italiano di Tecnologia, Via Enrico Melen, 83, 16152 Genoa, Italy 2DIBRIS, Università di Genova, , Via All'Opera Pia, 13, 16145 Genoa, Italy

* Correspondence: Monica Gori - monica.gori@iit.it 
Combining and integrating cues from different sensory channels is fundamental in developing a spatial representation of the environment. In the sighted population, the visual channel is essential in the spatial representation calibration; indeed, blind individuals show some impairments. One may compensate the vision loss to some degree by exploiting the associations between a movement and the consequent change in perceived auditory scene, known as audio-motor contingencies. The compensation extent is unclear, but evidence suggests that it depends on the amount of both visual and motor experience.

To quantify the extent of audio-motor compensation in relation to motor and audio-motor experience, we tested the spatial representation skills of a long-experienced early blind 5-a-side football player. We focused on auditory localization performance and sensitivity to an audio-motor contingency alteration. The study compared the player to groups of early blind (audio-motor experience without specific training) and sighted blindfolded people. We also tested an additional early blind individual without extensive audio-motor experience but who lost vision at an older age than the player, to control the effect of early visual experience alone.

Participants were tested on a set of steering tasks in auditory virtual reality (VR). In such tasks, participants would rotate a flying arrow towards an acoustic target. Rotations of the head or trunk controlled the arrow trajectory. Additionally, in some conditions, the relationship between movement and change of acoustic perceptual scene was altered to expose the participants' sensitivity to the audio-motor contingency alteration.

The early blind player performance was analyzed with classical univariate single-case statistics and a multivariate support vector machine classifier. Univariate analyses suggested that the early blind player's trunk motion is early blind-like. However, the multivariate classifier interpreted his overall performance as that of a sighted individual. The multivariate classifier labelled the visually experienced early blind's overall performance as early blind-like. We concluded that extensive audio-motor experience could compensate for early vision loss for what concerns the sensitivity to audio-motor contingency alterations. 
These results support the idea that adapted sports for visually impaired people are useful to improve their spatial representation and, consequently, their quality of life.

\section{Keywords: Audio-Motor Coordination; Spatial Orientation; Blind Football; Early Blindness; Motor Experience; Adapted Sport Practice.}

\section{INTRODUCTION}

The egocentric spatial representation is the ability to encode items' spatial features concerning oneself (Klatzky, 1998). Typically, its maturation requires visual information to be available until late childhood (Vasilyeva and Lourenco, 2012). Vision even plays a role in the auditory domain through cross-sensory calibration (Gori, 2015; Rohlf et al., 2020). Proof of vision's importance during development comes from the audio-spatial performance of those who lost vision early in life. There is no univocal definition for early blindness; authors have used age thresholds ranging from 2 to 13 years (Gougoux et al., 2004; Röder et al., 2004; Voss et al., 2004; Wan et al., 2010). Despite such differences, a number of studies indicate that early blind individuals have spatial deficits. For example, they are defective when it comes to estimating sounds' elevation, front-back discrimination (Voss, 2016) and absolute distance (Kolarik et al., 2016). However, they perform sighted-like or even better in estimating sounds' azimuth (Voss, 2016), suggesting some compensatory mechanism exists based on the available information. Scientific evidence has led researchers to hypothesize that the main egocentric spatial representation driver in the absence of vision may be the learned associations between motor actions and auditory scene changes, also known as audio-motor contingencies (Ashmead et al., 1998; Aytekin et al., 2008; Lewald, 2013, 2002). In the wake of these ideas, researchers have made several attempts to exploit naturally emerging (Honda et al., 2013) or artificially-evoked (Cuppone et al., 2018; Finocchietti et al., 2017; Sugano et al., 2010; TajaduraJiménez et al., 2012) audio-motor associations to modify various aspects of the egocentric spatial representation in both early and late blindness. Audio-motor training on visually impaired children was effective even one year after its execution (Cappagli et al., 2019). 
Such positive indications from audio-motor training paradigms make scientists wonder to which extent such paradigms can compensate for early vision loss. However, lab-based audio-motor training paradigms are by their nature limited in time, and if they are not included in rehabilitation settings, the life-long effect size is unknown. Moreover, they are usually focused on manipulating some specific abilities (Honda et al., 2013; Tajadura-Jiménez et al., 2012); therefore, they may exclude systemic interconnections, such as possible links between sensory information reliability and motor experience (Gori et al., 2012). Assessing the effect of variegate audio-motor training, possibly embedded in one's daily routine, would provide a better opportunity to understand the potential of audio-motor coordination. This is de-facto the description of an adapted sport. As a result, we decided to assess egocentric spatial representation after practicing over the long-term an adapted sport: football for the visually impaired, or 5-a-side football (see Gamonales, Muñoz-Jiménez, León-Guzmán, \& Ibáñez, 2018 for a review). In 5-aside football, visually impaired players become immersed in an acoustically rich environment while playing. The ball emits a rattling sound while moving; players must signal their presence with their voice, and sighted helpers placed behind the goal use their voice to drive players towards the goal. During practice, the players shall execute complex tasks, such as understanding the ball travel direction, understanding where they are in the playfield and where the other players are located, and coordinating their body movements to send the ball in the desired direction with the appropriate force (e.g., ball passes or shots on goal).

There is already some evidence that 5-a-side football promotes audio-motor skills related to egocentric spatial representation (Finocchietti et al., 2019; Mieda et al., 2019; Mieda and Kokubu, 2020; Velten et al., 2016, 2014). However, most of those studies are difficult to interpret in terms of egocentric spatial representation maturation. Some of them focus on kinematic behavior during football-related gestures, which is specific for the practice and unrelated to egocentric spatial representation (Finocchietti et al., 2019; Mieda and Kokubu, 2020). Some others find better audio-spatial performance for blind athletes, but no robust differences between sighted and blind non-athletes (Mieda et al., 2019; Velten et al., 2016, 
2014). Also, the experimental population composition plays a confounding role when it comes to interpreting the results. None of the previous studies explicitly state at what age people started playing 5a-side football, nor do they distinguish between early and late blindness. The latter misspecification is the most compelling sincebehavioral evidence suggests that audio-spatial abilities are more mature in the late blind than early blind populations (Voss, 2016). The practice of grouping early and late blind football players is unsurprising if one considers the players' population size. Even though epidemiologic information is limited, the picture drawn from the available surveys is that a very low percentage of blind people practice physical activity (Greguol et al., 2015; Haegele et al., 2017; Skaggs and Hopper, 1996; Wrzesińska et al., 2018). Moreover, early blind individuals are a minority among the blind population (GBD 2019 Blindness and Vision Impairment Collaborators on behalf of the Vision Loss Expert Group of the Global Burden of Disease Study, 2021). Consequently, early blind individuals with years of experience in practicing a sport are rare.

To overcome the scarceness of early blind people with extensive audio-motor experience, we used a single-case approach and focused on methodology to make them as interpretable and generalizable as possible. The most interpretable data for our situation is that early blind individuals expose a performance deficit concerning sighted individuals so that the 5-a-side football player performance will exist between the two. We have recently identified a similar pattern. We have developed a platform to assess egocentric spatial representation maturation in terms of (in)sensitivity to an audio-motor contingency alteration, i.e., alteration of the causal link between action and change of acoustic percept (Esposito et al., 2021). We have shown that early blind perform worse than sighted individuals in horizontal sound-alignment where trunk movements interfere with the head audio-motor contingency. Here, we investigate the hypothesis that an extensive audio-motor experience can compensate for early vision loss in maturing one's egocentric spatial representation. Starting from the assumption that long-term 5-a-side football practice provides extensive audio-motor training, we tested an early blind, long-experienced 5-a-side football player on the same platform used in the previous study and compared his performance to that of the early 
blind and sighted groups previously collected. To limit the confounding effect of early visual experience, we also tested an early blind individual with slightly more visual experience than the football player but who also had no extensive motor experience, as opposed to the 5-a-side football player. If extensive audio-motor experience could compensate for early vision loss, then the early 5-a-side football player behavior would be more like that of the people in the sighted group than that of the generic early blind group. Likewise, the visually experienced early blind participant's behavior would fall closer to the generic early blind group.

\section{METHODS}

\subsection{Participants}

One early blind, 5-a-side football player (male, age $=47$ years old) was recruited for the sake of this study. The early 5 -a-side football player had been practicing since 1985 . His data were compared to data previously collected from 21 individuals, 10 congenital blind ( 3 males, 7 females, age $=33.2 \pm 3.19$ years old) and 11 sighted individuals ( 6 males, 5 females, age $=31.27 \pm 3.92$ years old $)$ collected for another study. Since the early 5-a-side football player had vision until the age of 6 , we involved another blind individual in the study to help discern between the contribution of visual and motor experience. This latter blind individual had no extensive audio-motor experience, but he lost vision in the years of vision-based cross-sensory calibration (7 to 11 years) (Gori, 2015; Rohlf et al., 2020). None of the participants had been diagnosed with hearing or either cognitive impairments. Table 1 contains the clinical details of the participants' pathologies. All of them were enrolled from the local contacts of Liguria. Informed consent was obtained from all participants. The study followed the tenets of the Declaration of Helsinki and was approved by the ethics committee of the local health service (Comitato Etico, ASL 3, Genova). 


\begin{tabular}{|llllll|}
\hline & Gender & Age & Pathology & Blindness onset & Residual vision \\
\hline P1 & F & 32 & Retinopathy & Before birth & No vision \\
\hline P2 & F & 20 & Retinopathy & Before birth & Lights and shadows \\
\hline P3 & F & 29 & Retinopathy & Before birth & No vision \\
\hline P4 & M & 27 & Leber's amaurosi & Since birth & No vision \\
\hline P5 & F & 26 & Glaucoma and retinal detachment & Before birth & No vision \\
\hline P6 & M & 46 & Leber's disease & Before birth & No vision \\
\hline P7 & M & 52 & Unknown & Before birth & Lights and shadows \\
\hline P8 & F & 30 & Retinitis pigmentosa & Since birth & Lights and shadows \\
\hline P9 & F & 28 & Microphtalmia & Since birth & No vision \\
\hline P10 & F & 42 & Retinopathy & Since birth & No vision \\
\hline \hline P11 & M & 47 & Congenital glaucoma & 6 year-old & No vision \\
\hline \hline P12 & M & 59 & Uveitis & 11 year-old & Lights and shadows \\
\hline
\end{tabular}

Table 1: Clinical details of blind participants. The early blind 5-a-side football player is P11; the visually experienced early blind is $\mathrm{P} 12$

\subsection{Physical experimental setting}

The acoustic virtual environment used in this experiment was developed with the game engine Unity 3D. The resonance package (Google, 2018) provided the sounds' 3D rendering. Commercially available BOSE® over-ear headphones delivered the sounds. The headphones were connected to the HTC® VIVE head-mounted display (HMD) audio output channel. For the kinematic assessment, participants' head and trunk movements were tracked. The HMD itself tracked the participants' head rotations at a sampling rate of $90 \mathrm{~Hz}$, which is Unity 3D's main loop frequency. An LG® google nexus 4 smartphone used as a wireless inertial measurement unit tracked the trunk rotations by means of the app HyperIMU (ianovir, 2019), with a nominal sampling rate of $100 \mathrm{~Hz}$. Both sensors have a resolution of $0.1^{\circ}$. The incoming samples were asynchronously collected via a UDP socket; samples were then stacked and averaged at the frequency of $90 \mathrm{~Hz}$. To track the trunk, the experimenter put a custom-made harness on the participant; then, they placed the smartphone in a pocket lying approximately between the vertebrae T2 and T5. In the context of acoustic virtual reality, the HMD was blank, so its only purpose was the head tracking. The HTC VIVE HMD was preferred to a lighter device such as an IMU because its tracking system is reliable, accurate, and has been validated for scientific research (Le Chénéchal and Chatel-Goldman, n.d.; 
Niehorster et al., 2017; Verdelet et al., 2019). It also comes with default Unity 3D integration. The smartphone IMU was preferred at HTC Vive trackers to reduce the risk of trunk tracking loss for whatever reason. Tracking loss is known to dramatically reduce accuracy in Vive systems (Niehorster et al., 2017; Verdelet et al., 2019). However, IMUs drift during prolonged use. Therefore, recalibration was performed before starting a new condition and after approximately 10 trials to compensate for the drift effect. During the experiment, participants were seated and their trunk was free to rotate (Figure 1). The sitting position was chosen for safety reasons because there was no way to know whether a head-related audio-motor contingency alteration could impair participants' balance.

\subsection{Virtual experimental setting}

Our custom virtual reality platform defines a set of goal-directed steering tasks in first-person perspective, described as archery-like games. They are different parameterizations of one base task. The base task

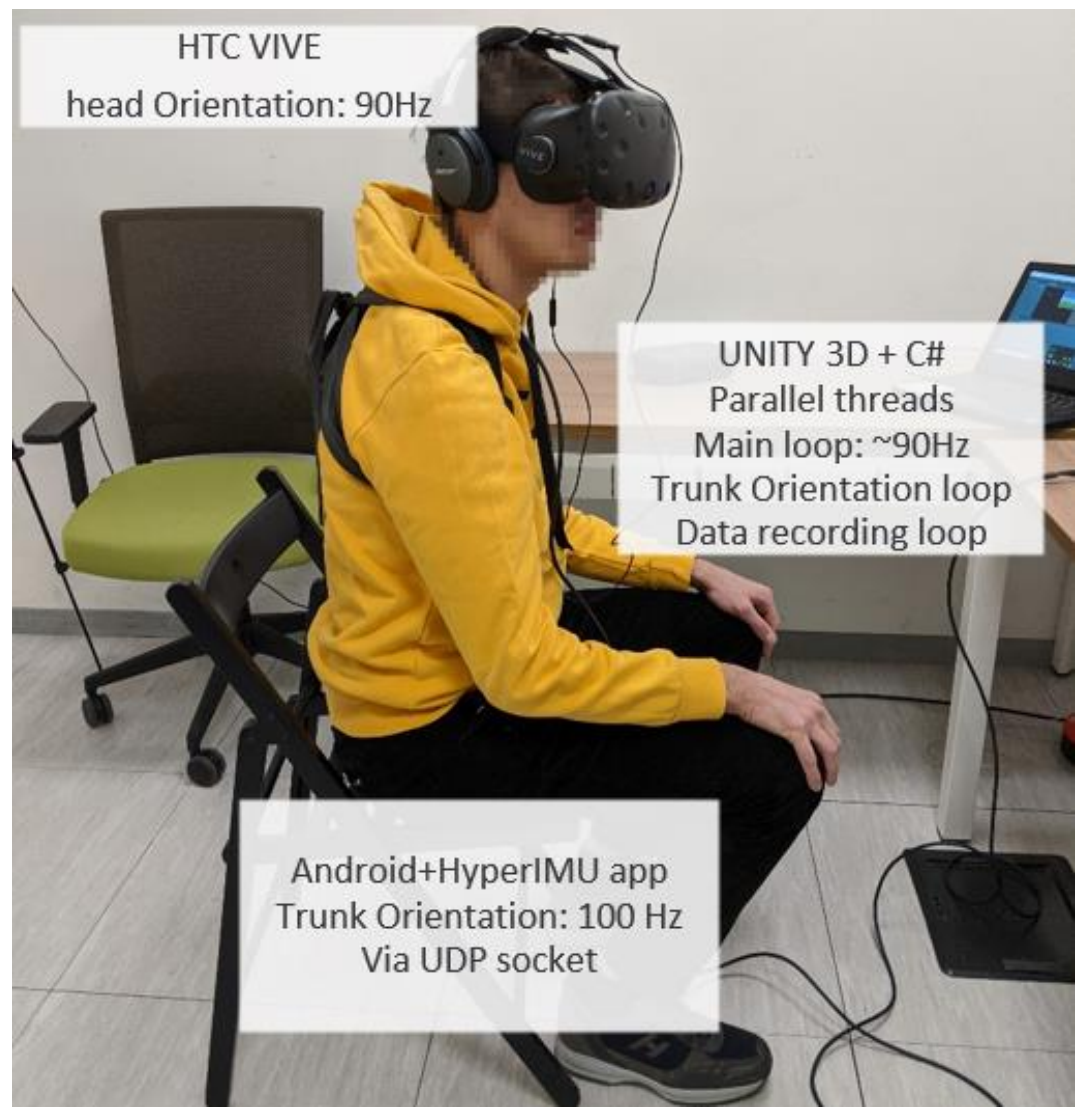

Figure 1: Experimental setting and tools specifications. The participant wears a blindfold and the HMD is blank. He/she can rest his/her back only during breaks and between conditions. 
consists of leading the arrow, which has an initial trajectory by-design of $15^{\circ}$ apart from the target, towards the target itself. The parameterizations differ from each other in terms of target position, arrow control and space-head-trunk audio-motor contingency state. The virtual environment's absolute reference frame is aligned to the participant's seat via the standard calibration phase for the HTC VIVE. The unit of measure of length is the unity unit (uu). Objects' sizes and distances have been designed to match the uu with the meter, so we use the meter to describe the spatial parameters. The arrow can be in two states only: loaded and shot. In the loaded state, the arrow appears at the origin of the virtual environment's absolute reference frame; it does not move, but it can rotate around the vertical axis. The transition from loaded to shot state is automatic. When the arrow orientation lies inside a trigger window for a period randomly chosen from 1 to $3 \mathrm{~s}$. The arrow, once shot, advances at a fixed velocity of $10 \frac{\mathrm{m}}{\mathrm{s}}$ in the horizontal plane. The target sound is intermittent pink noise with $5 \mathrm{~Hz}$ duty cycle and logarithmic spatial attenuation. It is distant $60 \mathrm{~m}$ from the starting point, and it can appear at three absolute angles: $-15^{\circ}, 0^{\circ}$, $+15^{\circ}$. Regardless of the task, trigger window and target centres are always $\pm 15^{\circ}$ apart. Figure $2 \mathrm{~A}$ displays the virtual environment, as well as the set of possible trigger-to-target geometrical configurations.

\subsection{Tasks description}

Among the available base task parameterizations, four ones were picked as experimental conditions. The conditions are defined according to two factors: "direction" and "coordination" (Figure 2). The factor "coordination" rules the combination of head and trunk movements leading the arrow direction in the virtual space. Its two levels are preserved and altered. The preserved level defines a simple soundalignment-by-head-pointing task. In this condition, the arrow rotation uniquely mimics the head yaw and the experimenter tells participants to move freely and focus on hitting the target. The altered level defines a sound-alignment-by-trunk-pointing task with voluntarily constrained head motion and altered audiomotor contingency. In this level, the arrow rotation mimics the trunk yaw, so a trunk-only rotation makes the participant perceive coherent self-rotation as if they had rotated the head in that direction. The insertion of a redundant head-trunk audio-motor contingency wants to degrade the reliability of audio- 
motor cues. Moreover, the experimenter explicitly asks the participant to immobilize their head as much as possible. This level aims to evaluate the participants' abilities when it comes to encoding audio-spatial information in a non-trunk-centred reference frame. The factor "direction" sets the relative initial position of the target concerning the arrow. Its two levels are central and lateral. In the central level, the target is in line with the participant's straight-ahead and the starting position of the arrow is $15^{\circ}$ to the side, randomly alternated between rightwards and leftwards. In the lateral level, the target lies at $15^{\circ}$ from the participant's straight-ahead, randomly alternating between rightwards and leftwards, and the starting position of the arrow is $0^{\circ}$. The participant starts with their head and trunk aligned to the straight-ahead direction. We distinguished between central and lateral targets because previous research on auditory localization in the horizontal plane by head-pointing showed different spatial performance in eccentric stimuli between sighted and blind people (Lewald et al., 2000; Makous and Middlebrooks, 1990; Occhigrossi et al., 2021).

Before starting with the trial, the participant is required to find the starting position. In the pre-trial phase, the arrow is loaded, and the participant is directed to place the arrow in the trigger window. The platform provides acoustic feedback that helps participants find the required starting orientation. It is an intermittent pure tone whose pitch is tuned by the angular distance between arrow and trigger window; the shorter the distance, the higher the pitch. The arrow shooting is announced by the interruption of the intermittent pure tone feedback and the reproduction of an arrow-shoot-like sound. The current study is not interested in the data collected in this first step. The second step, execution, is where the arrow moves and the task is accomplished. The trial end is notified by a pre-recorded sound clip of an arrow hitting a wall if the participant hits the target, or a windblown sound if they miss the target. 

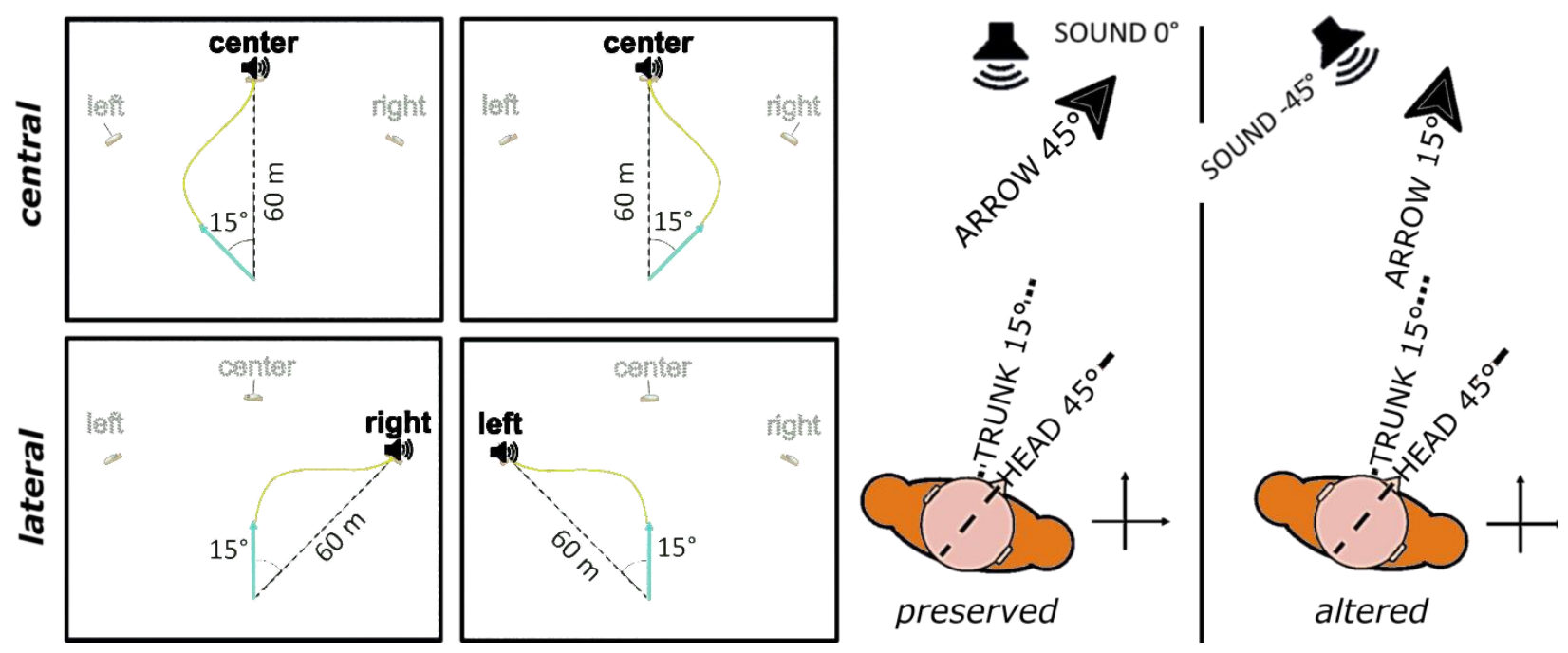

Figure 2: Schematic description of the experimental factors "direction" (A) and "coordination" (B). In panel A, two examples of possible arrow trajectories (yellow line) for each "direction" level are portrayed. The light blue arrow indicates the initial arrow trajectory. Targets with light grey names are inactive during the trial; the only active target is the one highlighted in black with the speaker icon on it. In panel B, spatial relationship between trunk, head, arrow and perceived sound source for each "coordination" level. Head yaw, trunk yaw and arrow direction are highlighted by, respectively, a dotted line and a dashed line.

\subsection{Experimental Procedure}

When the participant arrived, they were given the following instructions: "Imagine you are on an arrow.

Once shot, it will fly at a constant speed and you will control its direction only by moving a part of your body, as I will tell you. Your goal is to drive the arrow towards the sound you will hear, which will correspond to the target. There are three possible target positions." They were then blindfolded and led to haptically explore a scaled environment plastic model of the virtual platform. The experimenter guided the participant to use their finger to track four plausible arrow paths, two for each "direction" condition (e.g., yellow curved lines in figure 2A). A short training session was performed, involving a maximum of eight trials with preserved coordination and eight trials in altered coordination. This allowed the participant to familiarize themselves with the platform. For the training, only one "direction" level was chosen randomly, counterbalanced among participants.

Each experiment consisted of four blocks with one per condition executed in a randomized order, and counterbalanced among participants. Each block was made of twenty trials. Before proceeding with a block, the experimenter explained the corresponding requirements to the participant. In blocks with 
altered coordination, the experimenter helped participants who struggled at finding the starting point to keep their head in place until the starting point was reached. Breaks were allowed any time in accordance with participants' needs.

\subsection{Data Analysis}

\subsubsection{Data Pre-processing}

For each condition, four measures were computed: spatial accuracy, spatial precision, amount of head motion and amount of trunk motion. The accuracy is the median of the arrow distance from the target centre at the trial end (i.e. the final error). The precision is its interquartile range (IQR). The final error sign used to compute accuracy and precision identifies whether the arrow endpoint is beyond the target centre with respect to the starting direction: if so, it is positive, and if otherwise, it is negative. For both the head and trunk, the amount of motion was computed as the root mean square (RMS) of the third derivative (jerk) of the corresponding yaw signal. The jerk was chosen because it was the first derivative order that returned stationary signals according to the Dickey-Fuller stationarity test (Dickey and Fuller, 1979). Before differentiation, yaw signals were resampled at $90 \mathrm{~Hz}$ to compensate for sampling jitter and missing data points, then smoothed using an 18-samples moving average window. Trial-wise results were aggregated by means of the median. Overall, 16 response variables or features described each subject. . Data distributions for each feature were tested for normality by means of the Shapiro-Wilk test. The test rejected the normality hypothesis for many of them. Therefore, further analyses were carried on using nonparametric or parametric methods but robust to the gaussianity assumption violation. Data were analyzed using a univariate and a multivariate approach for assessing feature-specific and overall performances.

\subsubsection{Univariate Analysis}

Data were first compared in a group-wise fashion using the nonparametric Mann-Whitney U-test (hereafter-named U-test) to identify the features whose values significantly differed between early blind and sighted participants. Then, the Crawford-Garthwaithe Bayesian test (hereafter-named C-test) 
(Crawford and Garthwaite, 2007) was used to compare the early 5-a-side football player's values in each significant feature from the previous test to either the sighted or the early blind group. The C-test was most appropriate because it is robust to data non-gaussianity (Crawford and Garthwaite, 2012).

\subsubsection{Multivariate Analysis}

To gain insight into the early 5-a-side football player's global behavior, we decided to use a classification method rather than a standard statistical test. We implemented a linear kernel support vector machine classifier (SVM) (Ben-Hur and Weston, 2010) because it is a supervised classifier. Indeed, it does not assume any distribution for the underlying data and it can hold good performance for very small datasets (Vabalas et al., 2019). The SVM classifier was trained on early and sighted data using Leave-One-out Cross-Validation (LOOCV) (Webb et al., 2011) to limit overfitting. Specifically, for every training sample, a model was trained using z-standardized data from all training samples but the selected one; that sample was then labeled using that model. The model performance metrics were then computed from the labels distribution. The whole process was repeated for several values of the SVM's hyperparameter C. Supervised classification does not rely on the frequentist "null vs alternative hypothesis" logic. Therefore, to support our hypothesis, we selected the classifier with the highest possible sensitivity to the detection of an early blind individual, notwithstanding the higher risk of sighted participants' misclassification. In this way, we were able to minimize the chances of misclassifying an early blind participant. Accordingly, if the early 5-a-side football player behaved as an early blind individual, it would be classified as such.

The $\mathrm{C}$ value picked was 0.3 . We then classified the early 5 -a-side football player using a model trained on the whole training set.

\subsubsection{Software and data availability}

Kinematic data resampling, filtering and differentiation were made with the software MATLAB r2020a. Both univariate and multivariate analyses were made with the software R. Classification was made with the package CARET (Kuhn, 2020), C-tests were made with the package psycho (Makowski, 2018). The 
pre-processed dataset is available on the public repository Zenodo (Esposito, 2021). The code used for the analysis is available from the corresponding author on reasonable request.

\section{RESULTS}

\subsection{Univariate Analysis}

The U-test returned significant differences between early blind and sighted groups in the following features: accuracy for the central altered condition, $\mathrm{U}=86, \mathrm{p}=0.029$; precision for the central altered, $\mathrm{U}=85.5, \mathrm{p}=0.020$, and the lateral altered conditions, $\mathrm{U}=86, \mathrm{p}=0.029$; head RMS for the central altered condition, $\mathrm{U}=93, \mathrm{p}=0.006$; trunk RMS for the central altered, $\mathrm{U}=95, \mathrm{p}=0.004$, the central preserved,

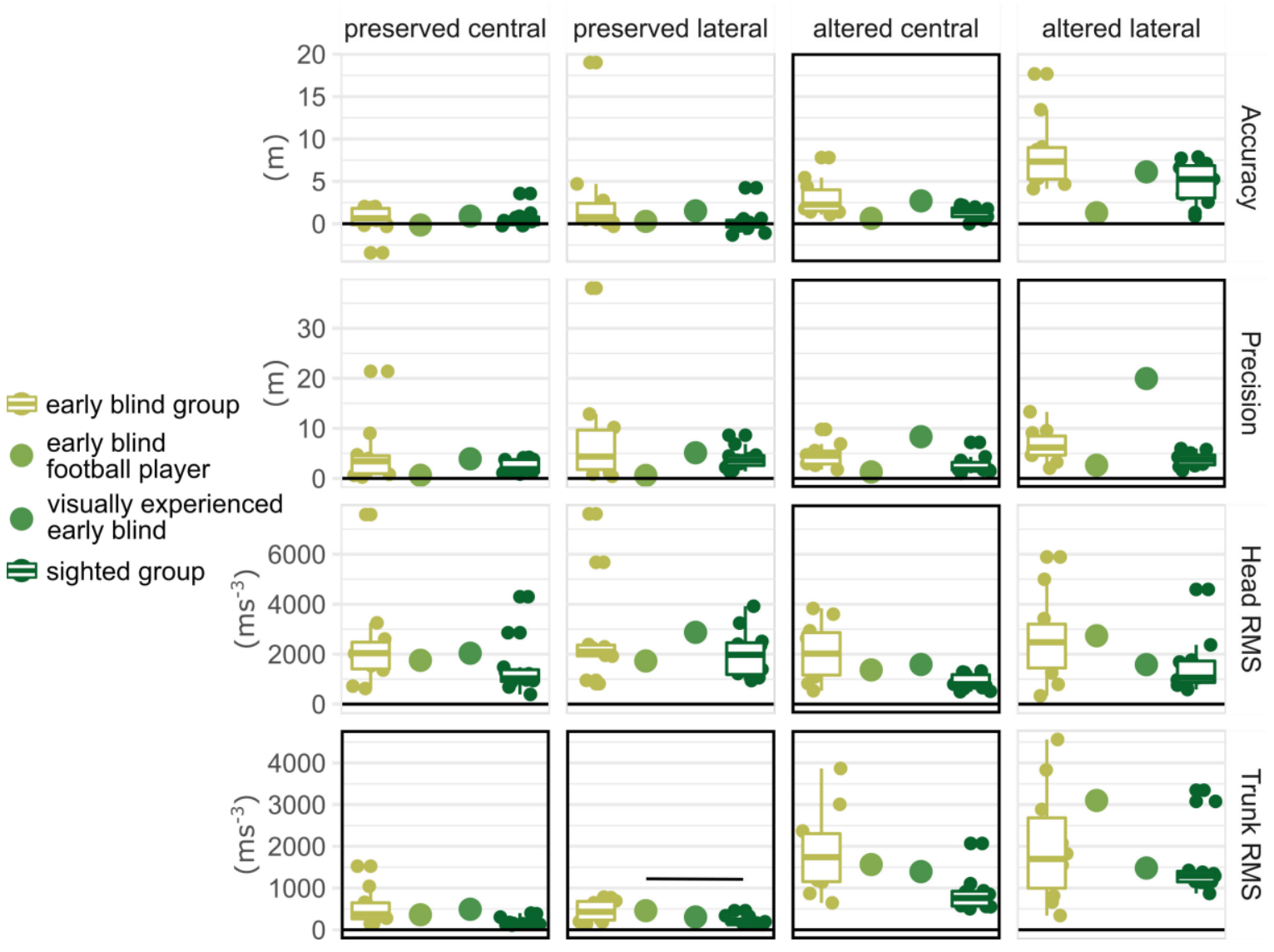

Figure 3: Data points for each measure, for each condition. Group distributions are described by boxplots. Data from early blind football player and visually experienced early blind are described by their corresponding data point. Thick outlines indicate the features where the U-test returned significant difference between early blind and sighted groups. Horizontal bars represent significant C-tests between early blind football player and given group. 


\begin{tabular}{|c|c|c|c|c|c|c|c|}
\hline & value & Percentile & group $\mathrm{M}$ & group SD & $\mathbf{p}$ & $\mathrm{Cl}$ & \\
\hline accuracy central altered & 0.6 & 13.3 & 3.1 & 2.2 & 0.158 & {$[0.015,0.348]$} & \multirow{7}{*}{$\underset{⿱ 亠 䒑}{\frac{\alpha}{4}}$} \\
\hline precision central altered & 1.3 & 7.9 & 4.7 & 2.4 & 0.105 & {$[0.003,0.266]$} & \\
\hline precision lateral altered & 2.6 & 12 & 6.6 & 3.3 & 0.148 & {$[0.008,0.328]$} & \\
\hline HeadRMS central altered & 1369.1 & 26.5 & 2088.9 & 1148.5 & 0.278 & {$[0.108,0.488]$} & \\
\hline TrunkRMS central preserved & 360.6 & 34.3 & 539.8 & 442.1 & 0.34 & {$[0.175,0.5]$} & \\
\hline TrunkRMS central altered & 1563.3 & 38.1 & 1868.3 & 1006.2 & 0.364 & {$[0.203,0.5]$} & \\
\hline TrunkRMS lateral preserved & 457.9 & 49.4 & 461.4 & 247.8 & 0.403 & {$[0.272,0.5]$} & \\
\hline
\end{tabular}

\begin{tabular}{|c|c|c|c|c|c|c|c|}
\hline accuracy central altered & 0.6 & 18.8 & 1.3 & 0.7 & 0.209 & {$[0.043,0.405]$} & \multirow{7}{*}{ 盇 } \\
\hline precision central altered & 1.3 & 22.7 & 2.7 & 1.8 & 0.243 & {$[0.069,0.443]$} & \\
\hline precision lateral altered & 2.6 & 20.9 & 3.8 & 1.4 & 0.228 & {$[0.06,0.426]$} & \\
\hline HeadRMS central altered & 1369.1 & 94.3 & 873.7 & 313.7 & 0.082 & {$[0,0.217]$} & \\
\hline TrunkRMS central preserved & 360.6 & 93.1 & 200.1 & 108.4 & 0.093 & {$[0.002,0.231]$} & \\
\hline TrunkRMS central altered & 1563.3 & 94.2 & 866.6 & 442.4 & 0.083 & {$[0.001,0.219]$} & \\
\hline TrunkRMS lateral preserved & 457.9 & 98.7 & 212 & 110.2 & $0.03 *$ & {$[0,0.106]$} & \\
\hline
\end{tabular}

Table 2: early blind player data point in absolute terms, relative to the refereence group, group mean (M) and standard deviation (SD), C-test $p$ value and credible interval. Both comparisons with the early blind and the sighted group are reported.

$\mathrm{U}=91, \mathrm{p}=0.010$, and the lateral preserved conditions, $\mathrm{U}=90, \mathrm{p}=0.013$. We used the 7 features identified above for the single-case investigation via individual C-tests. All the other features were not significant (all p-values >0.05).

None of the C-tests performed on the early 5-a-side football player versus the early blind group reached significance. Of the C-tests performed on the early 5 -a-side football player versus the sighted group, only the trunk RMS for the lateral preserved reached significance (single case datum value $=457.91 \mathrm{~ms}^{-3}$, sighted $\mathrm{M}=212.02 \mathrm{~ms}^{-3}$, sighted $\mathrm{SD}=110.18 \mathrm{~ms}^{-3}, \mathrm{p}=0.030$ ). Table 2 reports the complete list of C-test results. Univariate U-tests' results are available as supplementary material. Figure 3 reports data distributions together with a graphical description of univariate tests' significances.

\subsection{Multivariate Analysis}

The multivariate analysis did not focus on the extent to which single variables could discern among groups, as the univariate tests did, but rather aimed at distinguishing between the two groups as much as 
possible. LOOCV on the SVM classifier resulted in the training set having $100 \%$ sensitivity and $91 \%$ specificity for the early blind group: the model correctly classified all early blind participants and 10 out of 11 sighted participants. According to these metrics, if the football player behaved like the other early blind people, the model would classify him more likely as early blind than as sighted. In fact, the study classified the early 5-a-side football player as sighted.

The early 5-a-side football player's blindness onset was early but acquired: he lost vision at the age of 6 . Instead, the individuals in the early blind group were all congenital. Therefore, the visual experience collected by the early 5 -a-side football player is de facto a confounding factor. He may have been classified as sighted because of that visual experience and not because of the extensive audio-motor experience. An additional participant was inserted into the test set to probe the contribution of early vision. He too was acquired, having lost vision between 7 and 11 years of age, and he had no extensive audio-motor experience. He was tested on the same SVM classifier as the early 5-a-side football player, and classified as early blind.

\section{DISCUSSION}

In this case study, we tested the hypothesis that extensive motor experience can compensate for the role of vision in developing egocentric spatial representation. To do so, we tested an early blind, experienced 5-aside football player on an acoustic alignment task to test his audio-spatial abilities in the horizontal plane and his sensitivity to an audio-motor contingency alteration. We then used the sensitivity to the audiomotor contingency alteration as an indicator of egocentric spatial representation maturation. We assessed audio-spatial abilities and sensitivity to audio-motor alteration based on audio-spatial performance and motor behaviour. We compared the experienced 5-a-side football player performance to that of an early blind group without extensive motor experience, as well as to that of a sighted group.

Early blind and sighted people differed in seven out of sixteen features. In the standard sound-alignment by head-pointing task, early blind subjects moved the trunk more than sighted subjects in both travel 
directions (trunk RMS for central preserved and lateral preserved). With altered audio-motor contingency, the individuals in the early blind group were less precise in both travel directions than those in the sighted group (precision for central altered and lateral altered). Moreover, they were less accurate, and they moved the head and the trunk more than the sighted ones when targets were in front of them (accuracy, head RMS and trunk RMS for central altered).

Of the seven features where early blind and sighted people differed, none could discern between early 5a-side football player and the generic early blind group. Only the trunk RMS was significantly larger for the early 5-a-side football player than for the sighted population. The univariate analyses placed the early 5-a-side football player behavior between sighted and the generic early blind groups, slightly trending toward the latter. From these results, one would lean toward rejecting the initial hypothesis; however, the SVM classifier instead labelled the early 5-a-side football player as sighted. In addition, to clarify the contribution of early vision to the 5-a-sided football player overall performance, we classified (on the same trained classifier) another blind individual who lost vision later than the 5-a-side football player but did not undergo any extensive audio-motor training. If early vision were responsible for the model classifying the 5-a-side football player as sighted, the model should have classified this second individual as sighted as well. Instead, he was classified as early blind. We therefore concluded that the early blind player's egocentric spatial representation maturation, which we assessed as resilience to audio-motor contingency alterations, more likely depends on extensive audio-motor rather than early visual experience. Moreover, such maturation does not specifically contribute to any single ability. This result is in line with the previous findings about 5-a-side football's positive effects on several audio-spatial and motor abilities (Finocchietti et al., 2019; Mieda et al., 2019; Mieda and Kokubu, 2020; Velten et al., 2016, 2014). Moreover, it extends the current literature by showing for the first time that 5-a-side football's practice specifically benefits early blind individuals.

Given the 5-a-side football practice training, it is worth noticing that the egocentric spatial representation is a complex construct that involves knowing one's overall orientation (Klatzky, 1998). We probed this 
complex construct by testing the ability to align to a sound even after an audio-motor alteration affected the relationship between head-trunk rotations and egocentric orientation. However, even this ability depends on multiple aspects. It seems the overall egocentric orientation relies on a sort of summation of single body segments' orientations (Mergner et al., 1991). In turn, the single body segment orientations' goodness depends on the quality of the body representation, which in early blindness seems impaired (Imbiriba et al., 2006; Nava et al., 2014). Understanding at what level the 5-a-side football training operates is out of the present work's scope. Indeed, this study is limited to acknowledging the utility of the 5-a-side football practice as audio-motor training. Univariate analyses may have helped to unveil the audio-motor training effect more in detail, but they returned intermediate results. We are therefore limited to a speculative discussion. The speculation on the 5-a-side football effect starts from observing that most of the differences between early blind and sighted participants appear in the central altered condition. The mechanisms underlying such behavioral difference would require another study outside the current work; however, it is evident that early blind people struggled significantly more than those who were sighted when it came to aligning to targets placed straight-ahead. The early blind player instead did not significantly differ from the two groups in any feature for the central altered condition. We propose that the 5-a-side football practice may train the mental ability that facilitates, in sighted individuals, the alignment to sounds placed straight-ahead (Feierabend et al., 2019). Further investigation will test this hypothesis in detail.

Univariate analyses highlighted another important aspect. When aligning to a sideways target, the early 5a-side football player moved the trunk as much as the other early blind participants and significantly more than sighted participants. This finding indicates that, regardless of the egocentric spatial representation maturation, vision plays a significant role in choosing the motor strategy for coordinating head and trunk rotations. This process is the same strategy exposed by blind individuals in postural tasks (Alotaibi et al., 2016), and its pervasive use supports the idea that vision is fundamental in developing head-trunk coordination. 
A limit of this work is that it presents a single case study with small comparison samples, so that despite offering insight into 5-a-side football's effect on early blindness, its generalizability power is small. Nevertheless, the difficulty in recruiting such a specific population in Italy is still serious, as the number of experienced early blind 5-a-side football players is low. The football player under investigation here was indeed the only person we could find among our contacts. Luckily, most of the studies on 5-a-side football and audio-spatial or motor performances expose similar advantages from the practice. We therefore think that the cues obtained here are reliable for the work's scope.

To conclude, the practice of 5-a-side football resulted in useful audio-motor training to develop egocentric spatial representation. We hope that this work will foster 5-a-side football practice among early blind individuals as a way to improve their egocentric spatial representation, health, and social inclusion. These are three aspects where the margin for improvement remains large.

\section{ACKNOWLEDGEMENT}

The research is the results of MYSpace project, which has received funding from the European Research Council (ERC) under the European Union's Horizon 2020 research and innovation programme (grant agreement No 948349).

\section{AUTHOR CONTRIBUTIONS}

Davide Esposito: Conceptualization, Methodology, Software, Formal Analysis, Investigation, Data Curation, Writing - Original Draft, Visualization. Alice Bollini: Conceptualization, Methodology, Resources, Writing - Review \& Editing. Monica Gori: Conceptualization, Resources, Writing - Review $\&$ Editing, Supervision, Funding acquisition.

\section{DECLARATIONS OF INTEREST: NONE}




\section{REFERENCES}

Alotaibi, A.Z., Alghadir, A., Iqbal, Z.A., Anwer, S., 2016. Effect of absence of vision on posture. J. Phys. Ther. Sci. https://doi.org/10.1589/jpts.28.1374

Ashmead, D.H., Wall, R.S., Ebinger, K.A., Eaton, S.B., Snook-Hill, M.M., Yang, X., 1998. Spatial hearing in children with visual disabilities. Perception 27, 105-122. https://doi.org/10.1068/p270105

Aytekin, M., Moss, C.F., Simon, J.Z., 2008. A sensorimotor approach to sound localization. Neural Comput. 20, 603-635. https://doi.org/10.1162/neco.2007.12-05-094

Ben-Hur, A., Weston, J., 2010. A user's guide to support vector machines. Methods Mol. Biol. 609, 223239. https://doi.org/10.1007/978-1-60327-241-4_13

Cappagli, G., Finocchietti, S., Cocchi, E., Giammari, G., Zumiani, R., Cuppone, A.V., Baud-Bovy, G., Gori, M., 2019. Audio motor training improves mobility and spatial cognition in visually impaired children. Sci. Rep. 9, 3303. https://doi.org/10.1038/s41598-019-39981-x

Crawford, J.R., Garthwaite, P.H., 2012. Single-case research in neuropsychology: A comparison of five forms of t-test for comparing a case to controls. Cortex 48, 1009-1016. https://doi.org/10.1016/j.cortex.2011.06.021

Crawford, J.R., Garthwaite, P.H., 2007. Comparison of a single case to a control or normative sample in neuropsychology: Development of a Bayesian approach. Cogn. Neuropsychol. 24, 343-372. https://doi.org/10.1080/02643290701290146

Cuppone, A.V., Cappagli, G., Gori, M., 2018. Audio feedback associated with body movement enhances audio and somatosensory spatial representation. Front. Integr. Neurosci. 12, 37. https://doi.org/10.3389/fnint.2018.00037

Dickey, D.A., Fuller, W.A., 1979. Distribution of the Estimators for Autoregressive Time Series With a Unit Root. J. Am. Stat. Assoc. 74, 427. https://doi.org/10.2307/2286348 
Esposito, davide, Bollini, A., Gori, M., 2021. The link between blindness onset and audiospatial processing: testing audiomotor cues in acoustic virtual reality. https://doi.org/10.36227/TECHRXIV.14473047.V1

[dataset] Esposito, D., 2021. VRCR dataset clean v1.0.0. https://doi.org/10.5281/zenodo.4707477

Feierabend, M., Karnath, H.O., Lewald, J., 2019. Auditory Space Perception in the Blind: Horizontal Sound Localization in Acoustically Simple and Complex Situations. Perception 48, 1039-1057. https://doi.org/10.1177/0301006619872062

Finocchietti, S., Cappagli, G., Gori, M., 2017. Auditory Spatial Recalibration in Congenital Blind Individuals. Front. Neurosci. 11, 76. https://doi.org/10.3389/fnins.2017.00076

Finocchietti, S., Gori, M., Souza Oliveira, A., 2019. Kinematic Profile of Visually Impaired Football Players During Specific Sports Actions. Sci. Rep. 9, 1-8. https://doi.org/10.1038/s41598-019-47162Z

Gamonales, J.M., Muñoz-Jiménez, J., León-Guzmán, K., Ibáñez, S.J., 2018. 5-A-side football for individuals with visual impairments: A review of the literature. Eur. J. Adapt. Phys. Act. https://doi.org/10.5507/EUJ.2018.004

GBD 2019 Blindness and Vision Impairment Collaborators on behalf of the Vision Loss Expert Group of the Global Burden of Disease Study, 2021. Causes of blindness and vision impairment in 2020 and trends over 30 years, and prevalence of avoidable blindness in relation to VISION 2020: the Right to Sight: an analysis for the Global Burden of Disease Study. Lancet Glob. Heal. 9, e144-e160. https://doi.org/10.1016/S2214-109X(20)30489-7

Google, 2018. Resonance Audio SDK for Unity.

Gori, M., 2015. Multisensory Integration and Calibration in Children and Adults with and without Sensory and Motor Disabilities. Multisens. Res. 28, 71-99. https://doi.org/10.1163/22134808-00002478 
Gori, M., Tinelli, F., Sandini, G., Cioni, G., Burr, D., 2012. Impaired visual size-discrimination in children with movement disorders. Neuropsychologia $\quad 50, \quad 1838-1843$. https://doi.org/10.1016/J.NEUROPSYCHOLOGIA.2012.04.009

Gougoux, F., Lepore, F., Lassonde, M., Voss, P., Zatorre, R.J., Belin, P., 2004. Pitch discrimination in the early blind. Nature 430, 309-309. https://doi.org/10.1038/430309a

Greguol, M., Gobbi, E., Carraro, A., 2015. Physical activity practice among children and adolescents with visual impairment - Influence of parental support and perceived barriers. Disabil. Rehabil. 37, 327330. https://doi.org/10.3109/09638288.2014.918194

Haegele, J.A., Hodge, S.R., Kozub, F.M., 2017. Beliefs about physical activity and sedentary behaviors of adults with visual impairments. Disabil. Health J. 10, 571-579. https://doi.org/10.1016/j.dhjo.2017.03.008

Honda, A., Shibata, H., Hidaka, S., Gyoba, J., Iwaya, Y., Suzuki, Y., 2013. Effects of head movement and proprioceptive feedback in training of sound localization. Iperception. 4, 253-264. https://doi.org/10.1068/i0522

ianovir, 2019. HyperIMU.

Imbiriba, L.A., Rodrigues, E.C., Magalhães, J., Vargas, C.D., 2006. Motor imagery in blind subjects: The influence of the previous visual experience. Neurosci. Lett. 400, 181-185. https://doi.org/10.1016/j.neulet.2006.02.042

Klatzky, R.L., 1998. Allocentric and Egocentric Spatial Representations: Definitions, Distinctions, and Interconnections 1-17. https://doi.org/10.1007/3-540-69342-4_1

Kolarik, A.J., Moore, B.C.J., Zahorik, P., Cirstea, S., Pardhan, S., 2016. Auditory distance perception in humans: a review of cues, development, neuronal bases, and effects of sensory loss. Attention, Perception, Psychophys. 78, 373-395. https://doi.org/10.3758/s13414-015-1015-1 
Kuhn, M., 2020. caret: Classification and Regression Training.

Le Chénéchal, M., Chatel-Goldman, J., n.d. HTC Vive Pro time performance benchmark for scientific research.

Lewald, J., 2013. Exceptional ability of blind humans to hear sound motion: Implications for the emergence of auditory $\quad$ space. $\quad$ Neuropsychologia $\quad 51, \quad 181-186$. https://doi.org/10.1016/j.neuropsychologia.2012.11.017

Lewald, J., 2002. Opposing effects of head position on sound localization in blind and sighted human subjects. Eur. J. Neurosci. 15, 1219-1224. https://doi.org/10.1046/j.1460-9568.2002.01949.x

Lewald, J., Dörrscheidt, G.J., Ehrenstein, W.H., 2000. Sound localization with eccentric head position. Behav. Brain Res. 108, 105-125. https://doi.org/10.1016/S0166-4328(99)00141-2

Makous, J.C., Middlebrooks, J.C., 1990. Two-dimensional sound localization by human listeners. J. Acoust. Soc. Am. 87, 2188-2200. https://doi.org/10.1121/1.399186

Makowski, D., 2018. The Psycho Package: An Efficient and Publishing-Oriented Workflow for Psychological Science. J. Open Source Softw. 3, 470. https://doi.org/10.21105/joss.00470

Mergner, T., Siebold, C., Schweigart, G., Becker, W., 1991. Human perception of horizontal trunk and head rotation in space during vestibular and neck stimulation. Exp. Brain Res. 85. https://doi.org/10.1007/BF00229416

Mieda, T., Kokubu, M., 2020. Blind footballers direct their head towards an approaching ball during ball trapping. Sci. Rep. 10, 1-12. https://doi.org/10.1038/s41598-020-77049-3

Mieda, T., Kokubu, M., Saito, M., 2019. Rapid identification of sound direction in blind footballers. Exp. Brain Res. 237, 3221-3231. https://doi.org/10.1007/s00221-019-05670-4

Nava, E., Steiger, T., Roder, B., 2014. Both developmental and adult vision shape body representations. 
Sci. Rep. 4, 1-8. https://doi.org/10.1038/srep06622

Niehorster, D.C., Li, L., Lappe, M., 2017. The accuracy and precision of position and orientation tracking in the HTC vive virtual reality system for scientific research. Iperception. 8, 1-23. https://doi.org/10.1177/2041669517708205

Occhigrossi, C., Brosch, M., Giommetti, G., Panichi, R., Ricci, G., Ferraresi, A., Roscini, M., Enrico, V., Mario, P., 2021. Auditory perception is influenced by the orientation of the trunk relative to a sound source. Exp. Brain Res. https://doi.org/10.1007/s00221-021-06047-2

Röder, B., Rösler, F., Spence, C., 2004. Early Vision Impairs Tactile Perception in the Blind. Curr. Biol. 14, 121-124. https://doi.org/10.1016/j.cub.2003.12.054

Rohlf, S., Li, L., Bruns, P., Röder, B., 2020. Multisensory Integration Develops Prior to Crossmodal Recalibration. Curr. Biol. 30, 1726-1732.e7. https://doi.org/10.1016/j.cub.2020.02.048

Skaggs, S., Hopper, C., 1996. Individuals with visual impairments: A review of psychomotor behavior. Adapt. Phys. Act. Q. https://doi.org/10.1123/apaq.13.1.16

Sugano, Y., Keetels, M., Vroomen, J., 2010. Adaptation to motor-visual and motor-auditory temporal lags transfer across modalities. Exp. Brain Res. 201, 393-399. https://doi.org/10.1007/s00221-009-20473

Tajadura-Jiménez, A., Väljamäe, A., Toshima, I., Kimura, T., Tsakiris, M., Kitagawa, N., 2012. Action sounds recalibrate perceived tactile distance. Curr. Biol. https://doi.org/10.1016/j.cub.2012.04.028

Vabalas, A., Gowen, E., Poliakoff, E., Casson, A.J., 2019. Machine learning algorithm validation with a limited sample size. PLoS One 14, e0224365. https://doi.org/10.1371/journal.pone.0224365

Vasilyeva, M., Lourenco, S.F., 2012. Development of spatial cognition. Wiley Interdiscip. Rev. Cogn. Sci. 3, 349-362. https://doi.org/10.1002/wcs.1171 
Velten, M.C.C., Bläsing, B., Portes, L., Hermann, T., Schack, T., 2014. Cognitive representation of auditory space in blind football experts. Psychol. Sport Exerc. 15, 441-445. https://doi.org/10.1016/j.psychsport.2014.04.010

Velten, M.C.C., Ugrinowitsch, H., Portes, L.L., Hermann, T., Bläsing, B., 2016. Auditory spatial concepts in blind football experts. Psychol. Sport Exerc. 22, 218-228. https://doi.org/10.1016/j.psychsport.2015.08.010

Verdelet, G., Salemme, R., Desoche, C., Volland, F., Farnè, A., Coudert, A., Hermann, R., Truy, E., Gaveau, V., Pavani, F., 2019. Assessing Spatial and Temporal Reliability of the Vive System as a Tool for Naturalistic Behavioural Research, in: 2019 International Conference on 3D Immersion, IC3D 2019 - Proceedings. IEEE, pp. 1-8. https://doi.org/10.1109/IC3D48390.2019.8975994

Voss, P., 2016. Auditory Spatial Perception without Vision. Front. Psychol. 07, 1960. https://doi.org/10.3389/fpsyg.2016.01960

Voss, P., Lassonde, M., Gougoux, F., Fortin, M., Guillemot, J.-P., Lepore, F., 2004. Early- and Late-Onset Blind Individuals Show Supra-Normal Auditory Abilities in Far-Space. Curr. Biol. 14, 1734-1738. https://doi.org/10.1016/j.cub.2004.09.051

Wan, C.Y., Wood, A.G., Reutens, D.C., Wilson, S.J., 2010. Early but not late-blindness leads to enhanced $\begin{array}{llll}\text { auditory } & \text { perception. } & \text { Neuropsychologia } & 48,\end{array}$ https://doi.org/10.1016/j.neuropsychologia.2009.08.016

Webb, G.I., Sammut, C., Perlich, C., Horváth, T., Wrobel, S., Korb, K.B., Noble, W.S., Leslie, C., Lagoudakis, M.G., Quadrianto, N., Buntine, W.L., Quadrianto, N., Buntine, W.L., Getoor, L., Namata, G., Getoor, L., Han, X.J.J., Ting, J.-A., Vijayakumar, S., Schaal, S., Raedt, L. De, 2011. Leave-One-Out Cross-Validation, in: Encyclopedia of Machine Learning. Springer US, pp. 600-601. https://doi.org/10.1007/978-0-387-30164-8_469 
Wrzesińska, M., Lipert, A., Urzędowicz, B., Pawlicki, L., 2018. Self-reported physical activity using International Physical Activity Questionnaire in adolescents and young adults with visual impairment. Disabil. Health J. 11, 20-30. https://doi.org/10.1016/j.dhjo.2017.05.001 